\title{
A note on the thick subcategory theorem
}

\author{
Alain Jeanneret \\ University of Bern \\ CH-3012 Bern \\ jeanner@math-stat.unibe.ch \\ Peter S. Landweber * \\ Rutgers University \\ New Brunswick, New Jersey 08903 USA \\ landwebe@lagrange.rutgers . edu \\ Douglas C. Ravenel ${ }^{\dagger}$ \\ University of Rochester \\ Rochester, New York 14627 USA \\ drav@troi.cc.rochester.edu
}

July 14, 1994

\section{Introduction}

In this paper we will discuss an algebraic version (Theorem 1.6) of the thick subcategory theorem of Hopkins-Smith [HS] (Theorem 1.4). The former is stated as Theorem 3.4.3 in [Rav92], but the proof given there is incorrect. (A list of errata for [Rav92] can be obtained by email from the third author.)

First we recall the nilpotence theorem in its $p$-local version. Let $B P$ be the Brown-Peterson spectrum at the prime $p$, which satisfies:

$$
\pi_{*}(B P) \cong B P_{*} \cong \mathbf{Z}_{(p)}\left[v_{1}, v_{2}, \cdots\right],\left|v_{i}\right|=2\left(p^{i}-1\right) .
$$

Theorem 1.1 (Nilpotence theorem) [DHS88]

(i) Let $R$ be a p-local ring spectrum. The kernel of the BP Hurewicz homomorphism $B P_{*}: \pi_{*}(R) \longrightarrow B P_{*}(R)$ consists of nilpotent elements.

*Partially supported by the National Science Foundation

${ }^{\dagger}$ Partially supported by the National Science Foundation 
(ii) Let $f: F \longrightarrow X$ be a map from a p-local finite spectrum to an arbitrary spectrum. If $B P \wedge f$ is null homotopic, then $f$ is smash nilpotent; i.e. the $i$-fold smash product $f^{(i)}=f \wedge \cdots \wedge f$ is null for $i$ sufficiently large.

(iii) Let $\cdots \longrightarrow X_{n} \stackrel{f_{n}}{\longrightarrow} X_{n+1} \stackrel{f_{n+1}}{\longrightarrow} X_{n+2} \longrightarrow \cdots$ be a sequence of $p$-local spectra with $X_{n} c_{n}$-connected. Suppose that $c_{n} \geq m n+b$ for some $m$ and b. If $B P_{*} f_{n}=0$ for all $n$ then hocolim $X_{n}$ is contractible.

The Baas-Sullivan theory of bordism with singularities allows one to define ring spectra $K(n)$ and $P(n)$ for $0<n<\infty$ satisfying [Rav86]:

$$
\begin{gathered}
\pi_{*}(K(n)) \cong K(n)_{*} \cong \mathbf{F}_{p}\left[v_{n}, v_{n}^{-1}\right] \\
\pi_{*}(P(n)) \cong P(n)_{*} \cong \mathbf{F}_{p}\left[v_{n}, v_{n+1}, \cdots\right]
\end{gathered}
$$

as $B P_{*}$-algebras. We also set $P(0)=B P$ and $K(0)=H \mathbf{Q}$, the rational Eilenberg-Mac Lane spectrum. $K(n)$ is known as the $n^{\text {th }}$ Morava K-theory at the prime $p$. The following corollary of the nilpotence theorem will be proved in $\S 2$. This is stated in [Rav92] as Corollary 5.1.5, but again the proof given there is incorrect.

Corollary 1.2 Let $W, X$ and $Y$ be p-local finite spectra and $f: X \longrightarrow Y$. Then $W \wedge f^{(k)}$ is null homotopic for $k \gg 0$ if $K(n)_{*}(W \wedge f)=0$ for all $n \geq 0$.

Now let $\mathcal{C} \mathcal{P}_{0}$ be the homotopy category of finite $p$-local spectra and let $\mathcal{C P}{ }_{n} \subset \mathcal{C} \mathcal{P}_{0}$ be the full subcategory of $K(n-1)_{*}$-acyclics. In [Rav84] it was shown that the $\mathcal{C} \mathcal{P}_{n}$ fit into a sequence:

$$
\cdots \subset \mathcal{C P}{ }_{n+1} \subset \mathcal{C P} \mathcal{P}_{n} \subset \cdots \subset \mathcal{C P} \mathcal{P}_{0}
$$

Moreover all the inclusions are strict [Mit85].

Definition 1.3 A full subcategory $\mathcal{C}$ of $\mathcal{C} \mathcal{P}_{0}$ is thick if:

(i) An object weakly equivalent to an object in $\mathcal{C}$ is in $\mathcal{C}$.

(ii) If $X \longrightarrow Y \longrightarrow Z$ is a cofibration in $\mathcal{C} \mathcal{P}_{0}$ and two of $\{X, Y, Z\}$ are in $\mathcal{C}$ then so is the third.

(iii) A retract of an object in $\mathcal{C}$ is in $\mathcal{C}$.

Corollary 1.2 is the form of the nilpotence theorem needed to prove the thick subcategory theorem (see $\S 5.3$ of [Rav92]):

Theorem 1.4 (Thick subcategory theorem) If $\mathcal{C}$ is a thick subcategory of $\mathcal{C P} \mathcal{P}_{0}$, then there exists an integer $k$ such that $\mathcal{C}=\mathcal{C} \mathcal{P}_{k}$. 
Before we state an algebraic version of Theorem 1.4 let us fix some notation. Let $\mathcal{B} \mathcal{P}_{0}$ be the abelian category of $B P_{*}(B P)$-comodules finitely presented as $B P_{*}$-module [Lan76]. A typical object in $\mathcal{B P}_{0}$ is $B P_{*}(X)$ for $X$ in $\mathcal{C} \mathcal{P}_{0}$. We denote by $\mathcal{B} \mathcal{P}_{k}$ the full subcategory of $\mathcal{B} \mathcal{P}_{0}$ whose objects $M$ satisfy $v_{k-1}^{-1} M=0$ (we set $v_{0}=p$ ). Results of Johnson-Yosimura [JY80] (see also [Lan79] for a more algebraic proof) show that:

$$
\cdots \subset \mathcal{B P}_{k+1} \subset \mathcal{B P}_{k} \subset \cdots \subset \mathcal{B P}_{0} .
$$

Definition 1.5 Let $\mathcal{A}$ be an abelian category. A full subcategory $\mathcal{C}$ of $\mathcal{A}$ is thick if it satisfies the following condition:

If

$$
0 \longrightarrow M^{\prime} \longrightarrow M \longrightarrow M^{\prime \prime} \longrightarrow 0
$$

is a short exact sequence in $\mathcal{A}, M$ belongs to $\mathcal{C}$ if and only if $M^{\prime}$ and $M^{\prime \prime}$ belong to $\mathcal{C}$. (It means that $\mathcal{C}$ is stable under subobjects, quotient objects and extensions.)

The classification of the thick subcategories of $\mathcal{B P}_{0}$ is now the following; see $\S 3$ for the proof.

Theorem 1.6 (Algebraic thick subcategory theorem) If $\mathcal{C}$ is a thick subcategory of $\mathcal{B P}_{0}$, then there exists an integer $k$ such that $\mathcal{C}=\mathcal{B P}_{k}$.

Let us conclude the introduction with some remarks.

- Theorem 3.4.2 of [Rav92] is the analog of Theorem 1.6 stated in a different category, $C \Gamma$, which is defined in terms of $M U$ rather than $B P$.

- The $B P$-homology functor, $B P_{*}(\cdot): \mathcal{C P}_{0} \longrightarrow \mathcal{B P}_{0}$ sends the category $\mathcal{C P}_{k}$ into $\mathcal{B P}_{k}$. This comes from the fact [Rav84] that if $X \in \mathcal{C P}_{0}$ then

$$
K(n)_{*}(X)=0 \Longleftrightarrow v_{n}^{-1} B P_{*}(X)=0 .
$$

- Theorem 1.6 can be generalized to the abelian category of $P(n)_{*}(P(n))$ comodules, finitely presented over $P(n)_{*}$, which we denote by $\mathcal{P}(n)$. Similarly as for $\mathcal{B P}_{0}$ we can define the subcategories $\mathcal{P}(n)_{k}$ and prove the following.

Theorem 1.7 If $\mathcal{C}$ is a thick subcategory of $\mathcal{P}(n)$, then there exists an integer $k \geq n$ such that $\mathcal{C}=\mathcal{P}(n)_{k}$.

A further generalization of Theorem 1.6 can be obtained in the following setting. Let $E_{*}$ be a commutative $P(n)_{*}$-algebra such that $E_{*} \otimes_{P(n)_{*}}-$ is an exact functor on $\mathcal{P}(n)$. In [Lan76] the second author gave sufficient conditions for exactness. (The necessity of these conditions was shown by Rudyak in [Rud86].) Define

$$
E_{*}(E)=E_{*} \otimes_{P(n)_{*}} P(n)_{*}(P(n)) \otimes_{P(n)_{*}} E_{*} ;
$$


It can be made into a Hopf algebroid by extending the structure maps for $P(n)_{*}(P(n))$. Moreover $E_{*}(E)$ is a flat $E_{*}$-module because $P(n)_{*}(P(n))$ is a flat $P(n)_{*}$-module and if $N$ is a $E_{*}$-module then

$$
E_{*}(E) \otimes_{E_{*}} N \cong E_{*} \otimes_{P(n)_{*}}\left(P(n)_{*}(P(n)) \otimes_{P(n)_{*}} N\right) .
$$

If $M$ is an object of $\mathcal{P}(n)$ then $E_{*} \otimes_{P(n)_{*}} M$ is an $E_{*}(E)$-comodule via the $E_{*}$-extension of the composite:

$$
\begin{aligned}
M \longrightarrow P(n)_{*}(P(n)) \otimes_{P(n)_{*}} M & \\
\downarrow & \stackrel{\longrightarrow}{\longrightarrow} E_{*}(E) \otimes_{E_{*}}\left(E_{*} \otimes_{P(n)_{*}} M\right) .
\end{aligned}
$$

Let $\mathcal{E}$ be the category whose objects are $E_{*} \otimes_{P(n) *} M$ with $M \in \mathcal{P}(n)$ and morphisms $E_{*} \otimes f: E_{*} \otimes M_{1} \longrightarrow E_{*} \otimes M_{2}$ with $f: M_{1} \longrightarrow M_{2}$ in $\mathcal{P}(n)$; then $\mathcal{E}$ is an abelian category equipped with an exact functor:

$$
E_{*} \otimes_{P(n)_{*}}-: \mathcal{P}(n) \longrightarrow \mathcal{E} .
$$

The image of the subcategory $\mathcal{P}(n)_{k}$, written $\mathcal{E}_{k}$, satisfies:

$$
\cdots \subset \mathcal{E}_{k+1} \subset \mathcal{E}_{k} \subset \cdots \subset \mathcal{E}_{n}=\mathcal{E} .
$$

We are no longer claiming that the inclusions are strict. The thick subcategories of $\mathcal{E}$ can be described as follow:

Theorem 1.8 If $\mathcal{C}$ is a thick subcategory of $\mathcal{E}$, then there exists an integer $k \geq n$ such that $\mathcal{C}=\mathcal{E}_{k}$.

It should be emphasized that under the above assumption on $E_{*}$, the functor $E_{*} \otimes_{P(n)_{*}} P(n)_{*}(\cdot)$ is a homology theory [Lan76] taking its values in the category $\mathcal{E}$ as far as finite spectra are concerned.

\section{The proof of Corollary 1.2}

Let $D: \mathcal{C P}_{0} \longrightarrow \mathcal{C P}_{0}$ be the anti-equivalence induced by the SpanierWhitehead duality [Ada74]. If $X \in \mathcal{C} \mathcal{P}_{0}$ and $Y$ is any spectrum, the graded group $[X, Y]_{*}$ is isomorphic to $\pi_{*}(D X \wedge Y)$. We say that the maps $f: \Sigma^{n} X \longrightarrow$ $Y$ and $\widehat{f}: S^{n} \longrightarrow D X \wedge Y$ are adjoint if they correspond to each other under the above isomorphism of groups. In particular the adjoint of the identity $X \longrightarrow X$ is a map $e: S^{0} \longrightarrow D X \wedge X$. Recall that $X^{(i)}$ is a notation for the $i$-fold smash product $X \wedge \cdots \wedge X$.

Set $R=D W \wedge W$, a ring spectrum whose unit is $e$ and whose multiplication is the composite 


$$
R \wedge R=D W \wedge W \wedge D W \wedge W \stackrel{D W \wedge D e \wedge W}{\longrightarrow} D W \wedge S^{0} \wedge W=R .
$$

The map $f: X \longrightarrow Y$ is adjoint to $\widehat{f}: S^{0} \longrightarrow D X \wedge Y$ and $W \wedge f$ is adjoint to the composite

$$
S^{0} \stackrel{\widehat{f}}{\longrightarrow} D X \wedge Y \stackrel{e \wedge D X \wedge Y}{\longrightarrow} R \wedge D X \wedge Y,
$$

which we denote by $g$. Set $F=R \wedge D X \wedge Y$. The map $W \wedge f^{(i)}$ is adjoint to the composite

$$
S^{0} \stackrel{g^{(i)}}{\longrightarrow} F^{(i)}=R^{(i)} \wedge D X^{(i)} \wedge Y^{(i)} \longrightarrow R \wedge D X^{(i)} \wedge Y^{(i)},
$$

the latter map being induced by the multiplication in $R$.

We want to show that $W \wedge f^{(k)}$ is null for large $k$; by adjointness it suffices to prove that $g^{(k)}$ is null for large $k$. The second statement of Theorem 1.1 implies that we only need to show that $B P \wedge g^{(i)}$ is null for large $i$, so we can take $k$ to be an appropriate multiple of $i$. Let $T_{i}=R \wedge D X^{(i)} \wedge Y^{(i)}$ and let $T$ be the direct limit of

$$
S^{0} \stackrel{g}{\longrightarrow} T_{1} \stackrel{T_{1} \wedge \widehat{f}}{\longrightarrow} T_{2} \stackrel{T_{2} \wedge \widehat{f}}{\longrightarrow} T_{3} \longrightarrow \cdots .
$$

The desired conclusion will follow from showing that $B P \wedge T$ is contractible.

At this point we need to use the theory of Bousfield classes. Recall that the Bousfield class of a spectrum $X$ (denoted $\langle X\rangle$ ) is the collection of spectra $Z$ for which $X \wedge Z$ is not contractible. In [Rav84] it was shown that

$$
\langle B P\rangle=\langle K(0)\rangle \vee\langle K(1)\rangle \vee \cdots \vee\langle K(n)\rangle \vee\langle P(n+1)\rangle .
$$

By assumption, $K(n) \wedge T$ is contractible for all $n$. Therefore it suffices to show that $P(m) \wedge T$ is contractible for large $m$.

Since we are concerned only with finite spectra, we have for large enough $m$ :

$$
\begin{aligned}
K(m)_{*}(W \wedge f) & =K(m)_{*} \otimes_{\mathbf{F}_{p}} H_{*}\left(W \wedge f ; \mathbf{F}_{p}\right) \\
P(m)_{*}(W \wedge f) & =P(m)_{*} \otimes_{\mathbf{F}_{p}} H_{*}\left(W \wedge f ; \mathbf{F}_{p}\right) .
\end{aligned}
$$

Our hypothesis implies that both of these homomorphisms are trivial, so the smash product $P(m) \wedge T$ is contractible as required.

\section{The proof of Theorem 1.6}

The proof of Theorem 1.6 is a consequence of the filtration theorem of Landweber, namely 
Theorem 3.1 [Lan73] Each object $M \in \mathcal{B P}_{0}$ has a filtration

$$
0=M_{s} \subset \cdots \subset M_{1} \subset M_{0}=M
$$

in the category $\mathcal{B P}_{0}$, so that for $0 \leq i \leq s-1$ the quotient $M_{i} / M_{i+1}$ is stably isomorphic to $B P_{*} / I_{n_{i}}$ in $\mathcal{B} \mathcal{P}_{0}$, where $I_{n_{i}}=\left(p, v_{1}, \cdots, v_{n_{i}-1}\right)$ are invariant prime ideals of $B P_{*}$. (Stably isomorphic means isomorphic after a dimension shift.)

For $M \in \mathcal{B P}_{0}$ define $\operatorname{Spec}(M)=\left\{m \geq 1: v_{m-1}^{-1} M=0\right\} \bigcup\{0\}$ (set as usual $v_{0}=p$ ). If $M \neq 0$ then $\operatorname{Spec}(M)$ is a finite subset of $\mathbf{N}$ and is of the form:

$$
\operatorname{Spec}(M)=\left\{0,1, \cdots, N_{M}\right\}
$$

with $N_{M} \geq 0$.

Let $\mathcal{C}$ be a thick subcategory of $\mathcal{B} \mathcal{P}_{0}$. Define an integer $k$ by:

$$
\bigcap_{M \in \mathcal{C}} \operatorname{Spec}(M)=\{0,1, \cdots, k\} .
$$

From the definition of $k$, one has $\mathcal{C} \subset \mathcal{B P}_{k}$ and $\mathcal{C} \not \subset \mathcal{B P}{ }_{k+1}$. Let $M$ in $\mathcal{C}$ be such that

$$
v_{k-1}^{-1} M=0 \text { and } v_{k}^{-1} M \neq 0,
$$

and let

$$
0=M_{s} \subset \cdots \subset M_{1} \subset M_{0}=M
$$

be a Landweber filtration of $M$. As $\mathcal{C}$ is thick and $M \in \mathcal{C}$, all the $M_{i}$ 's belong to $\mathcal{C}$ as well as all the quotients $M_{i} / M_{i+1} \cong B P_{*} / I_{n_{i}}$.

Localization being an exact functor, all the $v_{k-1}^{-1} M_{i}$ are null and hence $v_{k-1}^{-1} M_{i} / M_{i+1} \cong v_{k-1}^{-1} B P_{*} / I_{n_{i}}=0$. Therefore

$$
n_{i} \geq k \text { for } 0 \leq i \leq s-1 .
$$

On the other hand, $v_{k}^{-1} M \neq 0$ implies the existence of a $j$ for which $v_{k}^{-1} B P_{*} / I_{n_{j}} \neq 0$, which forces

$$
n_{j} \leq k \text { for some } j, 0 \leq j \leq s-1 .
$$

From (3.2) and (3.3) we obtain that $n_{j}=k$ for some $j, 0 \leq j \leq s-1$, hence $B P_{*} / I_{k} \in \mathcal{C}$. Now it is fairly easy to prove by induction that $B P_{*} / I_{k+l} \in \mathcal{C}$ for all $l \geq 0$. Consider the exact sequence in $\mathcal{B P}_{0}$

$$
0 \longrightarrow B P_{*} / I_{k+l} \stackrel{v_{k+l}}{\longrightarrow} B P_{*} / I_{k+l} \longrightarrow B P_{*} / I_{k+l+1} \longrightarrow 0
$$


where the first morphism is multiplication by $v_{k+l}$. The subcategory $\mathcal{C}$ being thick, $B P_{*} / I_{k+l} \in \mathcal{C}$ implies $B P_{*} / I_{k+l+1} \in \mathcal{C}$.

We are now ready to show the inclusion $\mathcal{B P}_{k} \subset \mathcal{C}$. Let $N$ be an object in $\mathcal{B P}_{k}$ and $0=N_{s} \subset \cdots \subset N_{1} \subset N_{0}=N$ be a Landweber filtration of $N$. We have seen that $v_{k-1}^{-1} N=0$ implies $n_{i} \geq k$ for all $0 \leq i \leq s-1$ with, as usual, $n_{i}$ such that $N_{i} / N_{i+1} \cong B P_{*} / I_{n_{i}}$. By downward induction on $i$ we prove that $N_{i} \in \mathcal{C}$. This works as follows.

First $N_{s}=0 \in \mathcal{C}$. Second, the short exact sequence in $\mathcal{B P}_{0}$

$$
0 \longrightarrow N_{i+1} \longrightarrow N_{i} \longrightarrow B P_{*} / I_{n_{i}} \longrightarrow 0
$$

is such that $N_{i+1} \in \mathcal{C}$ (by the inductive assumption) and $B P_{*} / I_{n_{i}} \in \mathcal{C}$ as $n_{i} \geq k$. From the thickness of $\mathcal{C}$ we obtain that $N_{i} \in \mathcal{C}$. For $i=0$ we have $N \in \mathcal{C}$ and so $\mathcal{B P}_{k}=\mathcal{C}$, as required.

\section{References}

[Ada74] J. F. Adams. Stable Homotopy and Generalised Homology. University of Chicago Press, Chicago, 1974.

[DHS88] E. Devinatz, M. J. Hopkins, and J. H. Smith. Nilpotence and stable homotopy theory. Annals of Mathematics, 128:207-242, 1988.

[HS] M. J. Hopkins and J. H. Smith. Nilpotence and stable homotopy theory II. To appear in Annals of Mathematics.

[JY80] D. C. Johnson and Z. Yosimura. Torsion in Brown-Peterson homology and Hurewicz homomorphisms. Osaka Journal of Mathematics, 17:117-136, 1980.

[Lan73] P. S. Landweber. Associated prime ideals and Hopf algebras. Journal of Pure and Applied Algebra, 3:175-179, 1973.

[Lan76] P. S. Landweber. Homological properties of comodules over $M U_{*}(M U)$ and $B P_{*}(B P)$. American Journal of Mathematics, 98:591-610, 1976.

[Lan79] P. S. Landweber. New applications of commutative algebra to BrownPeterson homology. In P. Hoffman and V. Snaith, editors, Algebraic Topology, Waterloo 1978, pages 449-460, Springer-Verlag, New York, 1979.

[Mit85] S. A. Mitchell. Finite complexes with $A(n)$-free cohomology. Topology, 24:227-248, 1985.

[Rav84] D. C. Ravenel. Localization with respect to certain periodic homology theories. American Journal of Mathematics, 106:351-414, 1984. 
[Rav86] D. C. Ravenel. Complex Cobordism and Stable Homotopy Groups of Spheres. Academic Press, New York, 1986.

[Rav92] D. C. Ravenel. Nilpotence and periodicity in stable homotopy theory. Volume 128 of Annals of Mathematics Studies, Princeton University Press, Princeton, 1992.

[Rud86] Yu. B. Rudyak. Exactness theorems for the cohomology theories MU, $\mathrm{BP}$ and $\mathrm{P}(n)$. Mat. Zametki, 40:115-126, 1986. English translation in Math. Notes 40:562-569, 1986. 